\title{
Genome-Wide Identification and Characterization of the Cyclophilin Gene Family in the Nematophagous Fungus Purpureocillium lilacinum
}

\author{
Chenmi Mo ${ }^{\mathbb{D}}$, Chong Xie, Gaofeng Wang, Juan Liu, Qiuyan Hao, Xueqiong Xiao * and \\ Yannong Xiao * \\ The Provincial Key Lab of Plant Pathology of Hubei Province, College of Plant Science and Technology, \\ Huazhong Agricultural University, Wuhan 430070, China; mochenmi@webmail.hzau.edu.cn (C.M.); \\ xiechong@webmail.hzau.edu.cn (C.X.); jksgo@mail.hzau.edu.cn (G.W.); juandalin0704@163.com (J.L.); \\ 15738393165@163.com (Q.H.) \\ * Correspondence: xueqiongxiao@mail.hzau.edu.cn (X.X.); yannongxiao@mail.hzau.edu.cn (Y.X.)
}

Received: 17 May 2019; Accepted: 13 June 2019; Published: 18 June 2019

\begin{abstract}
Purpureocillium lilacinum has been widely used as a commercial biocontrol agent for the control of plant parasitic nematodes. Whole genome analysis promotes the identification of functional genes and the exploration of their molecular mechanisms. The Cyclophilin $(C Y P)$ gene family belongs to the immunophillin superfamily, and has a conserved cyclophilin-like domain (CLD). CYPs are widely identified in prokaryotes and eukaryotes, and can be divided into single- and multi-domain proteins. In the present study, 10 CYP genes possessing the CLD, named PlCYP1-P10, were identified from the genome of P. lilacinum strain 36-1. Those 10 PICYPs were predicted to have different cellular localizations in P. lilacinum. Phylogenetic and gene structure analysis revealed the evolutionary differentiation of CYPs between Ascomycotina and Saccharomycotina fungi, but conservation within the Ascomycotina fungi. Motif and gene structure distributions further support the result of phylogenetic analysis. Each $P l C Y P$ gene had a specific expression pattern in different development stages of P. lilacinum and its parasitism stage on eggs of Meloidogyne incognita. In addition, the 10 PlCYP genes exhibited different expression abundances in response to abiotic stresses, among which $P l C Y P 4$ was highly expressed at a high temperature $\left(35^{\circ} \mathrm{C}\right)$, while $P l C Y P 6$ was up-regulated under $5 \mathrm{mM}$ of $\mathrm{H}_{2} \mathrm{O}_{2}$ stress. Furthermore, the heterologous expression of PlCYP4 and PlCYP6 in Escherichia coli enhanced the cellular tolerance against a high temperature and $\mathrm{H}_{2} \mathrm{O}_{2}$. In summary, our study indicates the potential functions of $\mathrm{PlCYPs}$ in virulence and the stress response, and also provides a frame for further analysis of the $C Y P$ gene family in Ascomycotina fungi.
\end{abstract}

Keywords: Purpureocillium lilacinum; cyclophilin; phylogenetic analysis; expression pattern; abiotic stress

\section{Introduction}

Plant parasitic nematodes infect almost all cultivated plants worldwide and cause huge economic losses of up to $\$ 157$ billion per year [1]. It is difficult to discover nematode disease in fields owing to the small size of nematodes and the inconspicuous symptoms of infected plants [2]. The control of plant parasitic nematodes mainly depends on the application of nematicides, including carbofuran, ethoprophos, and aldicarb, while these pesticides have gradually been forbidden due to their adverse effects on the environment and human health. Therefore, biological control has become an alternative environment-friendly strategy for disease control. The nematophagous fungus, Purpureocillium lilacinum, is one of the most extensively tested fungi for controlling plant parasitic nematodes [3]. A number of studies have shown that $P$. lilacinum has a highly negative effect on the reproduction 
of nematodes. The application of P. lilacinum could effectively control second-stage juveniles, eggs, or egg masses of root-knot nematodes in pot experiments [4]. The combination of Syncephalastrum racemosum and P. linacinum can significantly decrease galls and nematodes in soil [5]. By soil application of P. lilacinum, the control efficacy against Meloidogyne javanica and Globodera pallida can reach above $70 \%$ [6]. So far, P. lilacinum has been registered as a biocontrol agent to control nematodes [7].

Genomes of four P. lilacinum strains have been sequenced [8-10]. According to the transcriptome analysis of P. lilacinum, a series of genes annotated as cyclophilins were found to display up-regulated expression when P. lilacinum infected nematodes [10]. Cyclophilins (CYPs) are a member of peptidyl prolyl cis-trans isomerases (PPIases), originally characterized as the receptor of an immunosuppressive drug cyclosporine A [11,12]. CYPs possess a highly conserved cyclophilin-like domain (CLD) and are divided into two types. One type is single-domain proteins that contain only the CLD, and the other is multi-domain proteins that harbor functional domains other than CLD, such as WD40, U-box, Leu Zipper, the RNA recognition motif (RRM), and tetratricopeptide repeats (TPRs) [13].

CYPs have been widely identified throughout prokaryotes (e.g., two in Escherichia coli) and eukaryote organisms such as fungi, nematodes, plants, animals, and humans [14-23]. CYPs play diverse roles in many cellular processes, including protein folding, cell morphogenesis, cell signaling, transcriptional regulation, RNA splicing, and the response to environmental stress [24-31]. However, the understanding of filamentous fungal CYPs and their potential functions is still preliminary. Studies of individual genes have revealed that CYPs are associated with virulence in filamentous fungi. The gene $B b C y p B$ with a signal peptide in the insect pathogenic fungus Beauveria bassiana contributes positively to virulence during the infection stage [32]. The hCYPA homologs in two plant pathogenic fungi, Magnaporthe grisea and Botrytis cinerea, were found to be closely related to pathogenicity [33,34].

The genome of P. lilacinum strain 36-1 has been sequenced [10]. In this study, we performed a genome-wide analysis of the CYP family members in P. lilacinum strain 36-1 (termed PICYPs), and further explored the phylogenetic relationship of PICYPs with other CYPs of Ascomycota fungi. We identified candidate PlCYPS associated with parasitism to nematodes, and also investigated the function of $P l C Y P S$ in response to abiotic stresses.

\section{Results}

\subsection{Ten CYP Genes Were Identified in P. lilacinum}

To identify CYP genes in P. lilacinum, the amino acid sequences of hCYPA (GenBank: AAI37059.1) were used to search against the P. lilacinum strain 36-1 genome and transcriptome database to obtain homologous sequences which were further confirmed by domain analysis [10]. In total, 10 non-redundant CYP genes were obtained and named PlCYP1-P10 (Table 1). To confirm the authenticity of acquired genes, the genomic DNA and cDNA of P. lilacinum 36-1 were used as templates, and they successfully amplified all of the genes (Figure 1A). The size of these 10 PlCYPs ranged from 162 to 627 amino acids, and the values of isoelectric points varied from 5.81 to 9.49 (Table 1). The prediction of subcellular localization revealed that PICYP1, 2, 4, 6, 8, and 10 were localized in the cytoplasm. PlCYP3 and PlCYP9 targeted the endoplasmic reticulum and mitochondria, while both PICYP5 and PICYP7 were shown to have nuclear localization signals, and were predicted to localize in the nucleus (Table 1). AtCYP59 and SpRct1 are orthologs of PICYP5 which localized in the nucleus and associated with transcriptional regulation $[35,36]$. Therefore, the localization of PICYP5 and PICYP7 was determined by the transient expression eGFP-tagged fusion proteins in Nicotiana benthamiana, suggesting that PlCYP5 and PICYP7 are localized in the nucleus (Figure 2). 
A

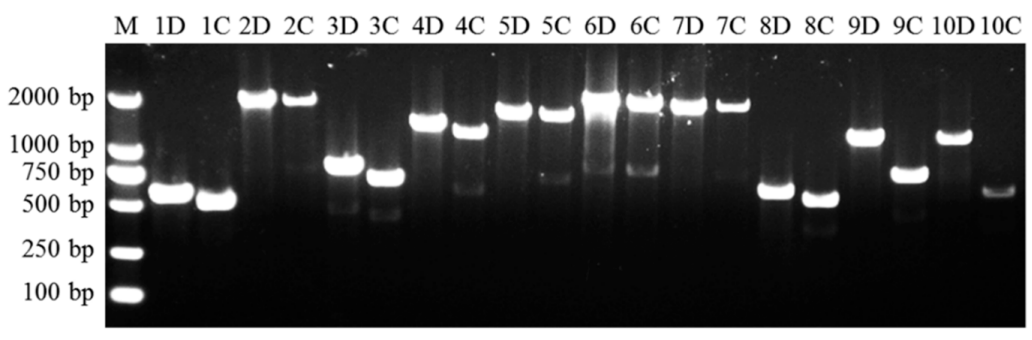

B

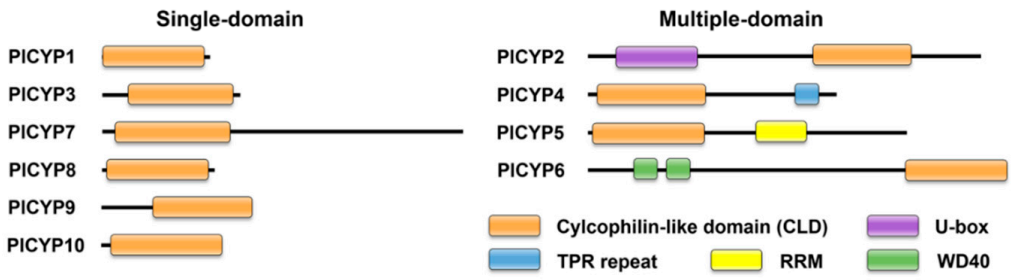

C

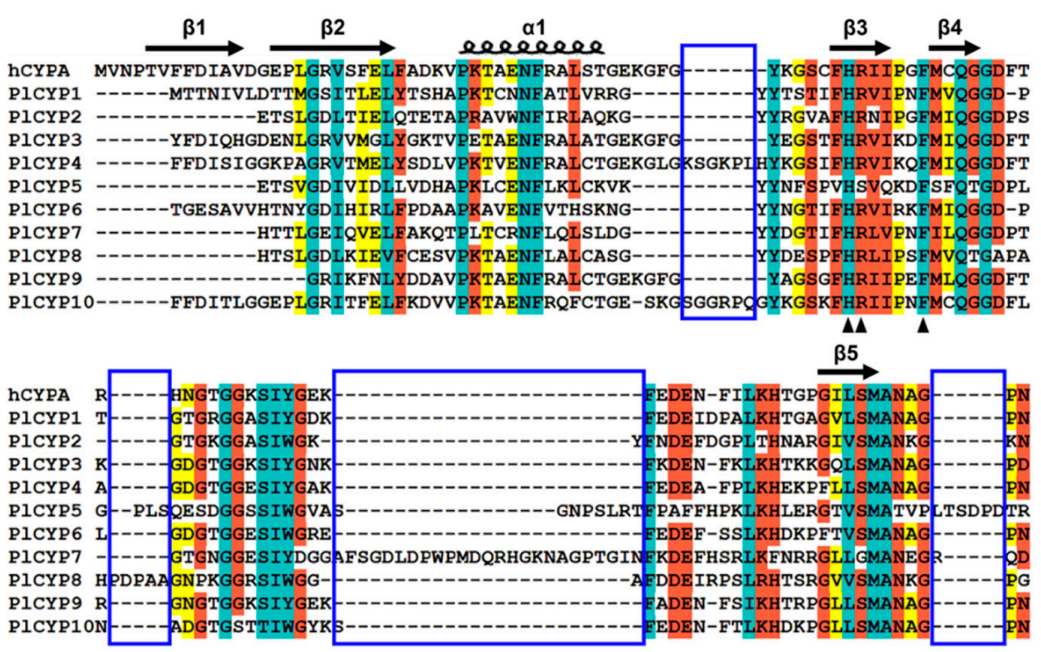

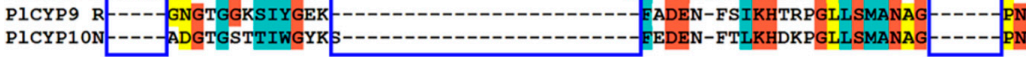

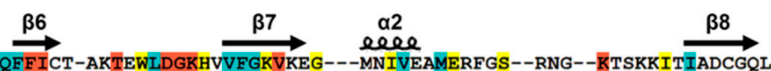

hCYPA TNGSQFFICT-AKTEWLDGKHVVFGKVKEG---MNTVEAMERFGS--RNG--KTSRKITIADCGQLE

P1CYP1 TNGSQFFITL-APIPWDGRHTIGEVIPPSPSTDAVIAKMEAVPTD-GSN--RPLNKILIKDVVV--

PICYP3 TNGSOFFITT-IITSWLDGRHVVFGEVLEG---YDIVDKIQNVKT--QPG-DKPEKTVKIVKSGE--

PICYP4 TNGSOFFITT-VPTPHLDGKHVVFGEVLNG---KSVVRQIENLQT--LSG-DKPTKDAVIADCGE--

P1CYP5 VAGSQFIITLGEDTDFLDGKAAIFGKVVEG---FDALEKINEAIVD-DKG--YPLIDIRIKHTVI--

P1CYP6 TNGSQFFITT-EKCPWLDGKHTIFGRATQG---FDVIQKIENART--YKE--KPEEDIKILNIDV--

P1CYP7 TNGSQFFFTL-DKTEELTGKNTMFGRVAGD--TIYNLAKIGESEVEPGSE--RPLYAVKIERIEI-

P1CYP8 TNGSQFFITF-DKASHLDGLNTIFGKVIGD-DSLATLAKMEKVEVD-KKS--RPKEPFRIEKVTI--

P1CYP9 TNGSQFFITT-VVTSWLDGRHVVFGEVADND-SLDVVKALEATGS--ASGSVKYNKRPTIVKSGE--

PICYP1OSNGSQFFITT-VPTPFLDGKHVVFGKVVDG---FDVVKKMEATKTG-YRGKDVENLDVVIAQCGE--

Figure 1. Members of the cyclophilin family in P. lilacinum (PICYPs). (A) PCR amplification to detect PlCYP genes. Lane $M$ is the DNA molecular weight marker, the numbers "1-10" in lanes 2-21 indicate genes PlCYP1-P10. The letter " $\mathrm{D}$ " indicates DNA as a template, and the letter " $\mathrm{C}$ " indicates cDNA as a template. "1D" indicates the use of the genomic DNA of P. lilacinum as a template to amplify the PlCYP1 gene. (B) Domains of PICYPs are shown in proportion to the length of sequences. The single-domain PICYPs are shown on the left side and the multi-domain PlCYPs are shown on the right side. (C) Multiple alignment of the conserved P. lilacinum cyclophilin-like domain (CLD) with the human CYPA (hCYPA). The secondary structures are displayed above the sequences. $\beta$ indicates the $\beta$ fold, and $\alpha$ indicates the $\alpha$ helix. Residues are shaded by different colors according to the conserved percent: cyan indicates $100 \%$; reddish orange means $80 \%$; and yellow indicates $60 \%$. Solid and hollow triangles denote residues predicted to have peptidyl prolyl cis-trans isomerases (PPIase) activity and a cyclosporin A binding site. The blue boxes represent the gaps between PlCYPs and hCYPA. 
Table 1. Characteristics of the Cyclophilin (CYP) gene family in Purpureocillium lilacinum strain 36-1.

\begin{tabular}{|c|c|c|c|c|c|c|}
\hline \multirow{2}{*}{ Gene Name } & \multirow{2}{*}{$\begin{array}{l}\text { Orthologs } \\
\text { in Strain } \\
\text { PLFJ-1 }\end{array}$} & \multirow{2}{*}{ ORF (bp) } & \multicolumn{3}{|c|}{ Deduced Polypeptide } & \multirow{2}{*}{$\begin{array}{c}\text { Predicted Subcellular } \\
\text { Localization }\end{array}$} \\
\hline & & & $\begin{array}{l}\text { Amino Acid } \\
\text { (aa) }\end{array}$ & $\begin{array}{c}\text { Molecular } \\
\text { Weight (kDa) }\end{array}$ & $\begin{array}{l}\text { Isoelectric } \\
\text { Point }\end{array}$ & \\
\hline$P l C Y P 1$ & XP_018182827.1 & 489 & 162 & 17.4 & 6.06 & Cytoplasm \\
\hline$P l C Y P 2$ & XP_018178542.1 & 1764 & 587 & 64.0 & 8.49 & Cytoplasm \\
\hline PlCYP3 & XP_018176208.1 & 627 & 208 & 22.8 & 7.89 & Endoplasmic reticulum \\
\hline PlCYP4 & XP_018179002.1 & 1119 & 372 & 40.3 & 5.81 & Cytoplasm \\
\hline PlCYP5 & XP_018178952.1 & 1437 & 478 & 54.8 & 5.93 & Nucleus \\
\hline PlCYP6 & XP_018179645.1 & 1884 & 627 & 70.2 & 6.49 & Cytoplasm \\
\hline$P l C Y P 7$ & XP_018177409.1 & 1623 & 540 & 60.2 & 5.98 & Nucleus \\
\hline PlCYP8 & XP_018177405.1 & 507 & 168 & 18.1 & 7.01 & Cytoplasm \\
\hline PlCYP9 & XP_018174420.1 & 684 & 227 & 24.6 & 9.49 & Mitochondria \\
\hline PlCYP10 & XP_018175923.1 & 549 & 182 & 19.8 & 6.29 & Cytoplasm \\
\hline
\end{tabular}

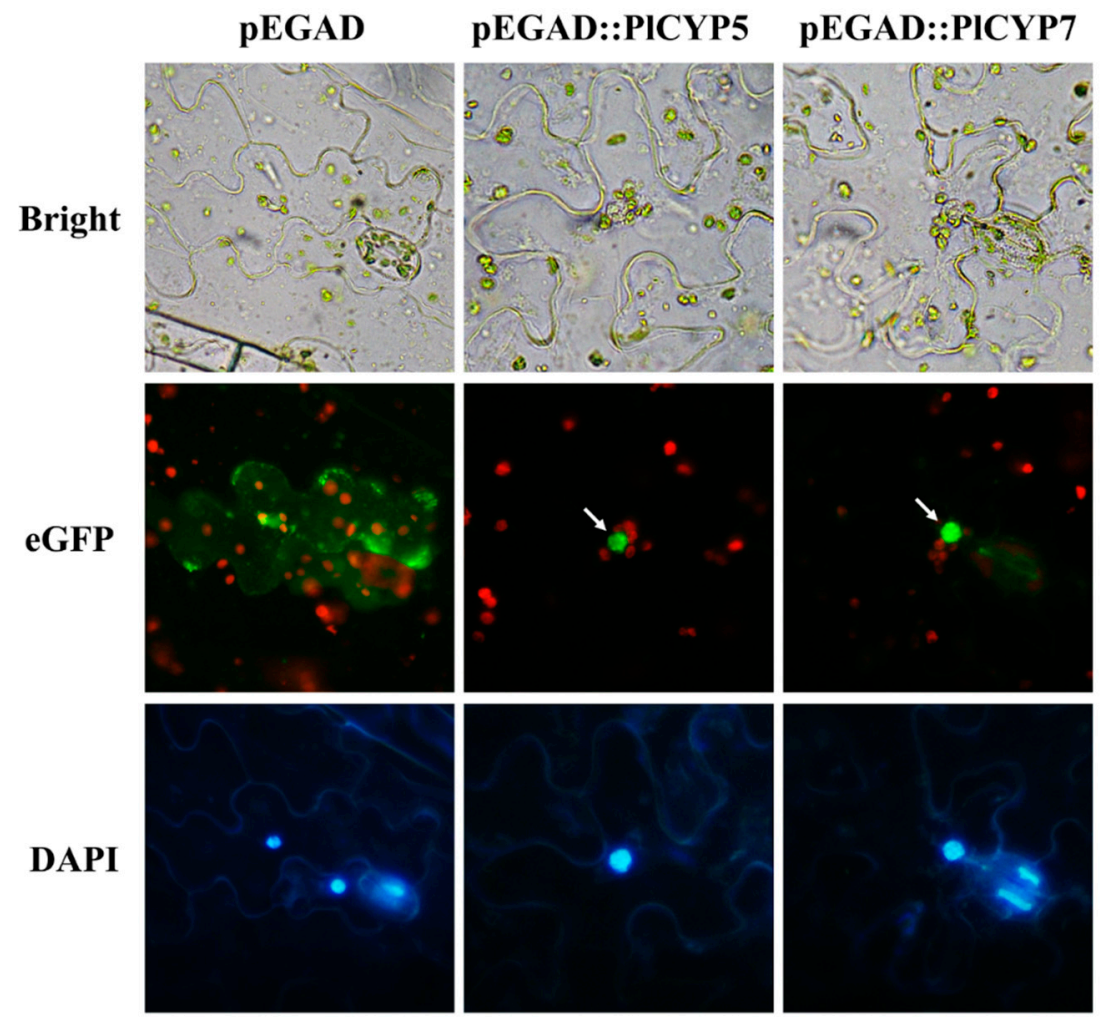

Figure 2. Subcellular localizations of PICYP5 and PICYP7 in Nicotiana benthamiana. Agrobacterium tumefaciens was used to transiently express eGFP::PICYP5 or eGFP::PlCYP7 fusion proteins in the leaves of $N$. benthamiana, and transient expression of eGFP was used as the control. Subcellular localizations of the fused proteins were visualized using fluorescence microscopy at $48 \mathrm{~h}$ after infiltration, and 4', 6-diamidino-2-phenylindole was used to stain the nucleus of epidermal cells before microscopic observation. The arrows indicate proteins localized in the nucleus.

\subsection{The PlCYPs Contain the CLD Domain with Residue Variation}

The ScanProsite analysis showed that, among the 10 PlCYPs, PlCYP1, 3, 7, 8, 9 and 10 are single-domain proteins that only contain the CLD, while PICYP2, 4, 5, and 6 possess additional domains. PICYP2 and PICYP6 contain a U-box and WD40 domain in the N-terminal of their sequences, while PICYP4 has a TPR domain in the C-terminal and PICYP5 possesses an RRM (Figure 1B).

In CYPs, CLD is the PPIase functional domain that contains many conserved residues, including H54, R55, F60, Q111, F113, W121, and H126 specific to hCYPA [37]. To obtain more sequence details, alignment of the CLD region was performed and the hCYPA, which represents the typical CYP, was used as the reference. The result indicated that sequences encoding the secondary structures of CLD in 
each PICYP were conserved (Figure 1C). However, the residues for PPIase activity and cyclosporin A binding were partially mutated in PICYPs such as PICYP5 and PICYP7 (Figure 1C, triangle). In addition, PlCYP4, 5, 7, 8, and 10 contained extra short sequences that hCYPA lacks, for which four gaps were shown by multiple alignment analysis (Figure 1C, blue box).

\subsection{Phylogenetic Analysis Showed Evolutionary Divergence of CYPs between Ascomycotina and Saccharomycotina Fungi}

To explore the evolutionary relationship within fungal CYPs, besides that of CYPs in P. lilacinum, sequences of 94 CYPs from different fungal species belonging to the Ascomycota phylum (Ascomycotina and Saccharomycotina) were collected, and used to construct a phylogenetic tree. As observed in Figure S1, the phylogenetic tree was divided into 10 groups (group A to J) based on sequence homology, and the PlCYPs were distributed in different groups. Eight pairs of CYPs from B. bassiana and P. lilacinum were clustered together, including PlCYP2/BbCYP8, PlCYP3/BbCYPB, PlCYP4/BbCYPD, PlCYP6/BbCYPE, $\mathrm{PlCYP7/BbCYP9,} \mathrm{PlCYP8/BbCYP3,} \mathrm{PlCYP9/BbCYPA,} \mathrm{and} \mathrm{PlCYP10/BbCYPH.} \mathrm{In} \mathrm{addition,} \mathrm{only} \mathrm{groups}$ G, H, and I had CYPs of Saccharomycotina fungi, but their CYPs were separated into the Ascomycotina fungal CYPs in these groups, which formed two independent branches (groups G1/G2, H1/H2 and I1/I2).

To further understand the clustering manner, all labels were tinted with different background colors, representing different predicted cellular localizations. It showed that the CYPs of the same predicted cellular localization tend to cluster together. Proteins predicted to target the mitochondria were distributed in group $\mathrm{H}$, and groups A and B only contained CYPs that were predicted to localize in the nucleus (Figure S1). Moreover, the multi-domain CYPs with similar domain architectures were also clustered together, including groups B, E, F, and I (Figure S1).

\subsection{The CYPs in Ascomycotina Fungi Display a More Complex Motif and Gene Structure Distribution than the CYPs in Saccharomycotina}

The MEME program was employed to analyze the conserved and potential motifs of all selected fungal CYPs. Twenty motifs were identified, and their distribution was displayed corresponding to the phylogenetic tree (Figure S2A). This showed that the CYPs in the same group have similar motifs (Figure S2B). The sequences of motifs were then annotated (Table S1), showing that six motifs (motif 1, 2, 3, 4, 5, and 7) are part of the CLD and existed in all groups except for group B, which lacks motifs 2 and 5 (Figure S2B). Moreover, some motifs exclusively appeared in a certain group of proteins. For example, only members of group B possess motif 6 (Figure S2B), motifs 9, 10, 12, 16, and 20 are only presented in group F, and motifs 14 and 15 only exist in group I (Figure S2B).

The gene structure analysis showed that intron numbers in coding sequences of the 10 PlCYPS vary from 0 to 5 . Similar to the motifs, the exon-intron distribution was diverse in different groups (Figure S2C). Additionally, the intron numbers are different for members in the same group. Proteins in group B had intron numbers that range from 0 to 3, whereas, groups $G, H$, and I which contain both Ascomycotina and Saccharomycotina fungal CYPs exhibited more complex intron numbers and lengths (Figure S2C). Nevertheless, CYPs clustered into pairs display similar distributions, such as PlCYP5/GzCYP8, PlCYP8/BbCYP3, and PlCYP3/BbCYPB.

\subsection{The PlCYP6 Gene Has Two Transcripts}

Among the $10 \mathrm{PlCYPs}, \mathrm{PlCYP6}$, whose coding product possesses the CLD and WD40 domain, has the longest length (Figure 1B). The comparison of the transcriptome and genome indicated that PICYP6 has two transcripts. To confirm this result, specific primers were used for amplification and sequencing, and two transcripts were obtained, transcript-a (T-a) and transcript-b (T-b) (Figure 3B). Thus, PlCYP6 has two introns within its coding region, and the second intron can either be spliced or not. In the case of $\mathrm{T}-\mathrm{a}$, the second intron is retained, and the translation process will end at the 
termination codon TAA, which is located in the second intron, otherwise, if the second intron is spliced, the translation process will end at the termination codon TAG (Figure 3A).

A

T-a

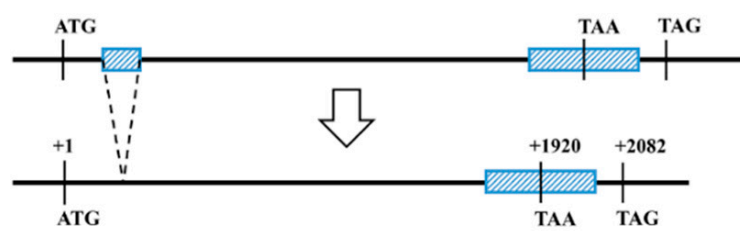

T-b

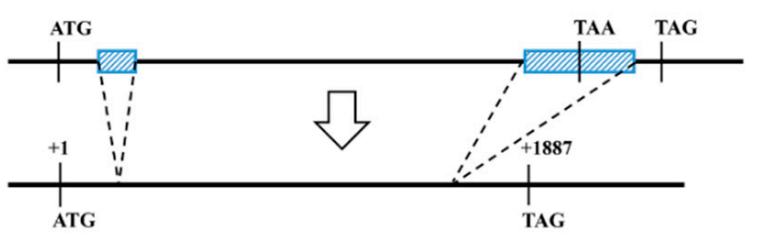

B

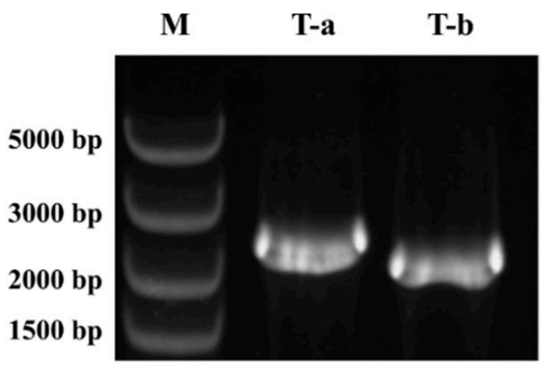

Intron

Figure 3. Alternative splicing of the PlCYP6 gene in P. lilacinum. (A) Diagram of the alternative splicing process, generating two transcripts (T-a and T-b). (B) PCR amplification of the two transcripts with specific primers using the cDNA of strain $36-1$ as a template.

\subsection{PlCYPs Exhibit Different Expression Patterns in Different Fungal Morphologies and at the Egg} Parasitic Stage

To overview the roles and activities of PICYPs across fungal developments, their expression levels were assessed using the real-time RT-PCR analyses of total RNAs prepared from conidia, germinating conidia, blastospores, vegetative hyphae, and aerial hyphae of P. lilacinum strain 36-1 (Figure 4A). The quantitative results showed that the 10 PlCYPs were ubiquitously expressed in all samples (Figure 4B). Compared with un-germinated conidia, all PlCYPs except PlCYP7 were significantly up-regulated after $8 \mathrm{~h}$ of germination, and two genes, PlCYP5 and PlCYP6, had significantly higher expressions in blastospores than in conidia (Figure 4B). In addition, compared with the hyphae in the vegetative growth stage, the expression levels of PlCYPs in aerial hyphae were almost the same, with only the expressions of PlCYP4 and PlCYP9 being significantly decreased (Figure 4B).

Considering that $P$. lilacinum is an egg-parasite fungus, the eggs of the nematode were inoculated with fungal conidia of P. lilacinum, and relative expressions of PlCYPS were investigated at different times after inoculation (hpi), with 0 hpi being used as the control. The results showed that the expressions of all 10 PlCYPs increased after the inoculation of eggs. Many genes, such as PlCYP3, 6, 8, and 9, exhibited significant expression at later stages of inoculation (Figure 5). In particular, PlCYP5 was significantly up-regulated at all time points with the highest expression at 6 hpi (Figure 5). 
A
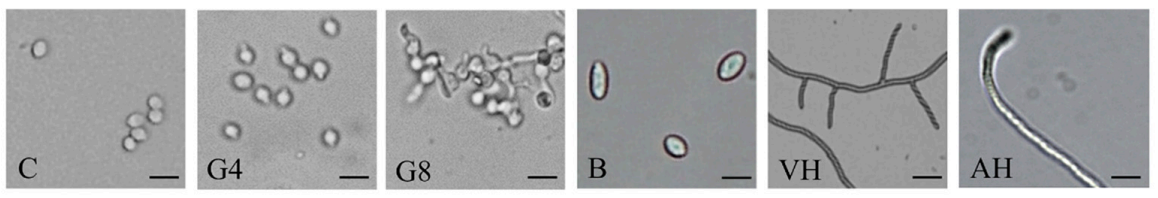

B
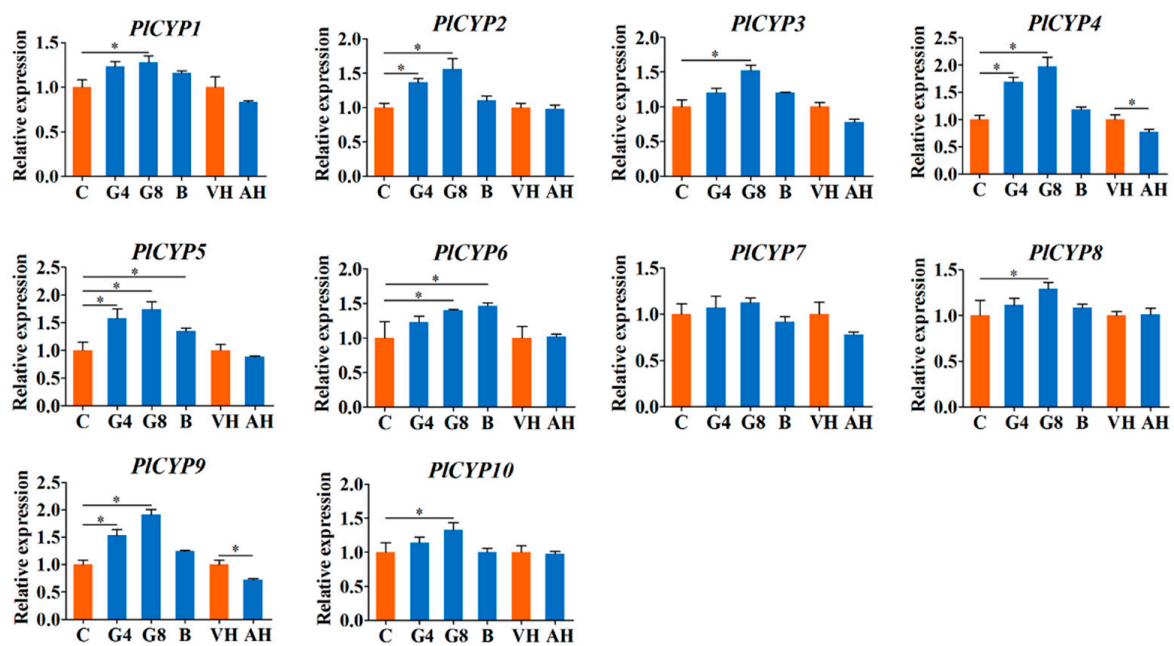

Figure 4. Expression levels of PlCYPs in different fungal morphologies. (A) Microscopic images of $P$. lilacinum at different growth stages. " $\mathrm{C}$ " indicates conidia, "G4" indicates conidia germinated for $4 \mathrm{~h}$, "G8" indicates conidia germinated for $8 \mathrm{~h}$, "B" indicates blastospores, "VH" indicates vegetative hyphae, and "AH" indicates aerial hyphae. Scale $=5 \mu \mathrm{m}$. (B) Relative expression levels of PlCYPS in conidia germination for $4 \mathrm{~h}$, germination for $8 \mathrm{~h}$ and blastospores versus conidia, and relative expression levels in aerial hyphae versus vegetative hyphae. The $\beta$-actin and tubulin genes were used as internal controls to normalize the data. The error bar represents the standard deviation (SD) of three replicates. ${ }^{*}$ denotes $p<0.01$ in the variance analysis.
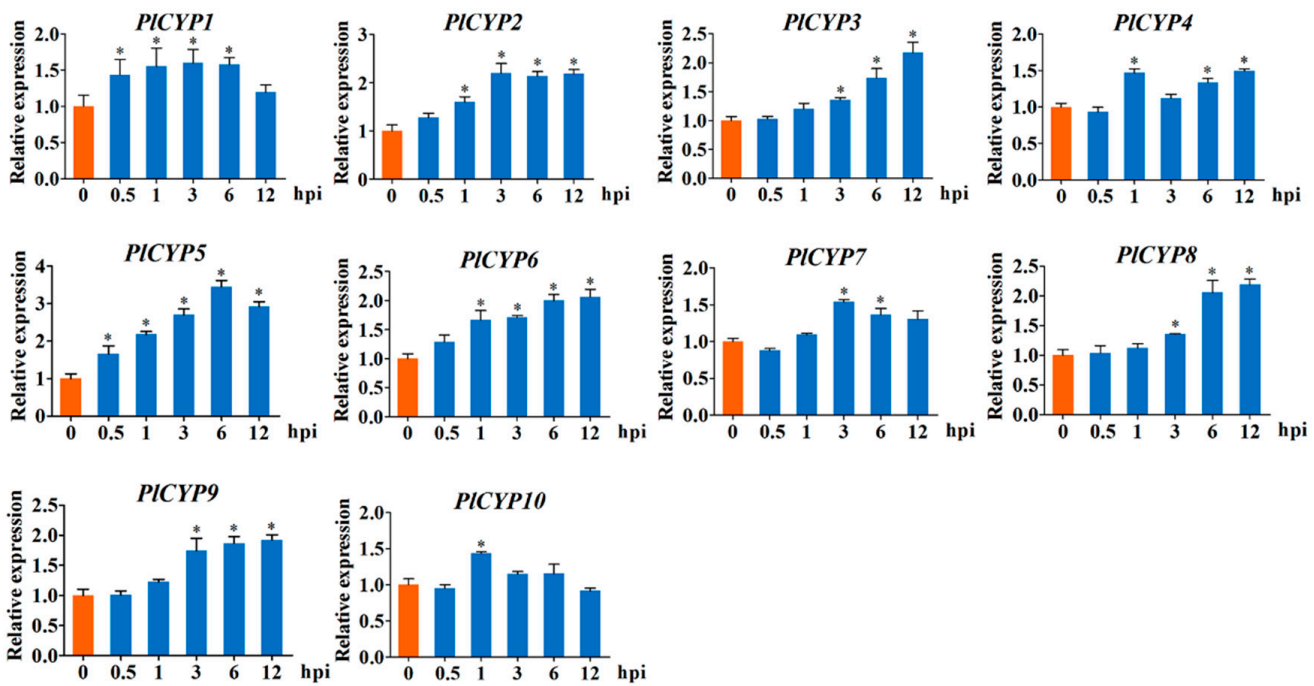

Figure 5. Expression levels of PlCYPs at the Meloidogyne incongnita parasitic egg stage. Relative expression levels of PlCYPs at $0.5,1,3,6$, and $12 \mathrm{~h}$ post inoculation (hpi) versus the control with no eggs. The $\beta$-actin and tubulin genes were used as internal controls to normalize the data. The error bar represents the SD of three replicates. ${ }^{*}$ denotes $p<0.01$ in the variance analysis. 


\subsection{The Expression of PICYP4 and PICYP6 Were Induced under High Temperature and $\mathrm{H}_{2} \mathrm{O}_{2}$ Stresses}

The mycelia were grown on solid medium for long-term stress and stimulated in liquid media for short-term stress. The expression levels of PlCYPs were analyzed by comparing similarities and differences between long-term and short-term stress response tests (Figure S3). As shown in Figure 6, there are differences between the two results. All PlCYPS were down-regulated when P. lilacinum grew on the plates containing $1 \mathrm{M} \mathrm{NaCl}$ and $1.2 \mathrm{M}$ sorbitol, whereas $P l C Y P 2,5$, and 6 were positively induced during the short-term response period (Figure 6). Nevertheless, two genes showed consistency in their expression levels. PlCYP6 showed the most active response to $\mathrm{H}_{2} \mathrm{O}_{2}$ both in the long-term and short-term assays, and the $\mathrm{PlCYP} 4$ maintained the highest expression in both assays after high temperature treatment (Figure 6).

A

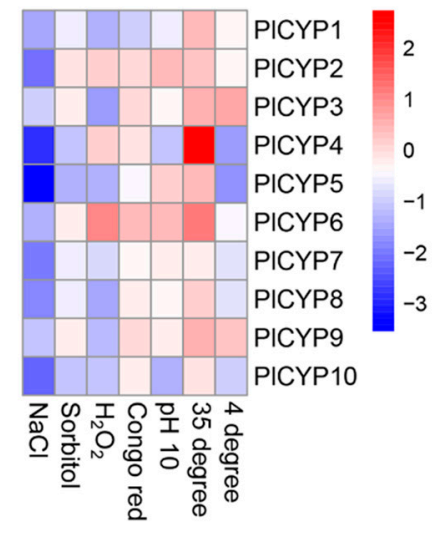

B

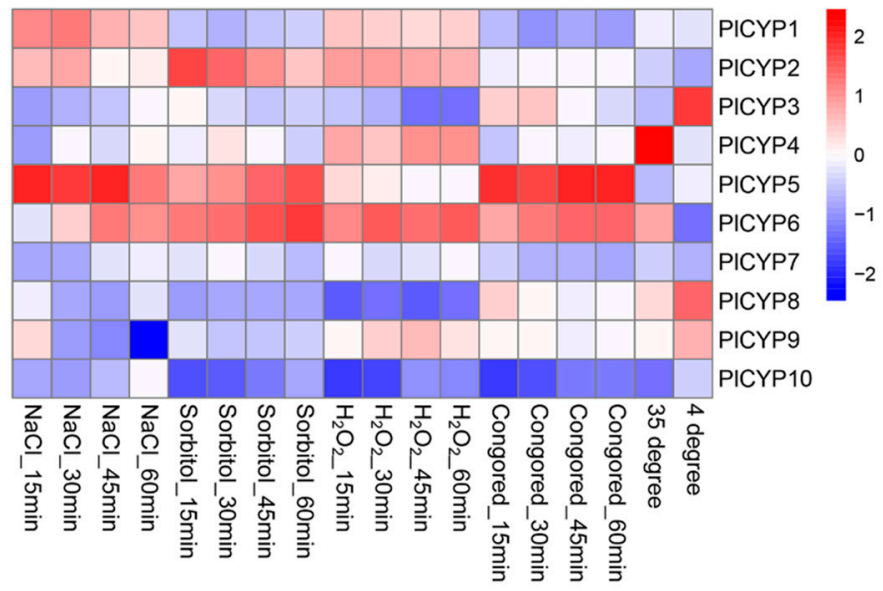

Figure 6. Expression of $P l C Y P s$ under different abiotic stresses. (A) Relative expression of $P l C Y P S$ on solid Czapek-Dox medium (CZM) media under different abiotic stresses, including $1 \mathrm{M} \mathrm{NaCl}, 1.2 \mathrm{M}$ sorbitol, $5 \mathrm{mM} \mathrm{H}_{2} \mathrm{O}_{2}, 0.15 \mathrm{mg} / \mathrm{mL}$ Congo red, alkaline stress ( $\mathrm{pH} 10$ ), and temperature stress ( 35 and $4{ }^{\circ} \mathrm{C}$ ). (B) Relative expressions of PlCYPs in liquid CZM media under the above-mentioned abiotic stresses. The fold changes of gene expressions were calculated versus the control by the $2^{-\Delta \Delta \mathrm{Ct}}$ method, and the logarithm $(\log 2)$ of those values was applied to generate the heat map.

\subsection{Heterologous Expressions of PlCYP4 and PlCYP6 in E. coli Enhance Tolerance towards Abiotic Stresses}

The expression results suggest that $P l C Y P 4$ and PlCYP6 have higher expressions in response to high temperature and the presence of $\mathrm{H}_{2} \mathrm{O}_{2}$, respectively (Figure 6). Therefore, heterologous expressions of PlCYPs in E. coli were determined based on the growth of E. coli on Luria-Bertani (LB) solid media supplemented with different abiotic stresses to detect whether PlCYP4 and PlCYP6 are involved in abiotic stress tolerance (Figure S4). Due to PlCYP6 having alternative splicing, heterologous expressions of transcripts T-a and T-b were both tested. The E. coli cultured on LB basal plates was used as the control, showing that the growth of recombinants and the wild type strain was similar (Figure S5). On the LB plate supplemented with $1 \mathrm{mM} \mathrm{H}_{2} \mathrm{O}_{2}$, both transcripts T-a and T-b of the PlCYP6 recombinants grew, but the control and PlCYP4 recombinant were unable to grow (Figure S5). On LB plates containing $600 \mathrm{mM} \mathrm{NaCl}, 800 \mathrm{mM}$ sorbitol, or LB medium for which the $\mathrm{pH}$ was adjusted to 10, tolerance was not displayed by any of the recombinants (Figure S5).

In order to quantify the growth rate, the liquid culture method was adopted under the conditions of $\mathrm{H}_{2} \mathrm{O}_{2}$ and a high temperature. The liquid cultures were diluted to appropriate concentrations and spread on LB media to count the colony numbers. As expected, in liquid culture containing $\mathrm{H}_{2} \mathrm{O}_{2}$, the growth of E. coli recombinants expressed T-a and T-b better than in the wild type control (Figure 7A). In addition, after being treated at $70{ }^{\circ} \mathrm{C}$ for $20 \mathrm{~min}$, the colonies of the PlCYP4 recombinant were significantly more numerous than with the control (Figure 7B). 
A

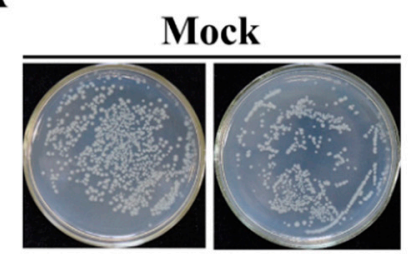

pET-28a 28a-PICYP4

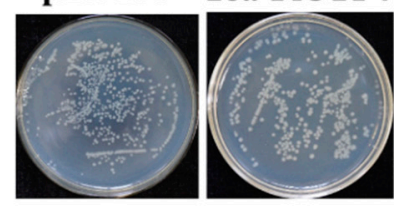

28a-PICYP6 28a-PICYP6

(T-a)

(T-b)

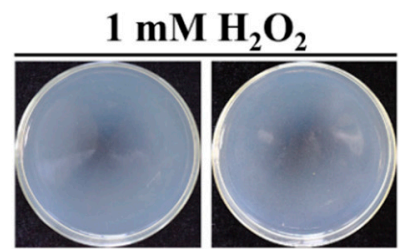

pET-28a 28a-PICYP4

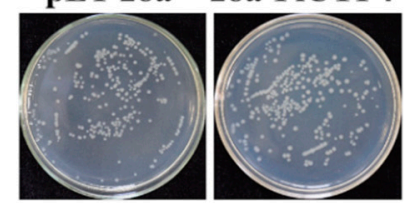

28a-PICYP6 28a-PICYP6

(T-a)

(T-b)
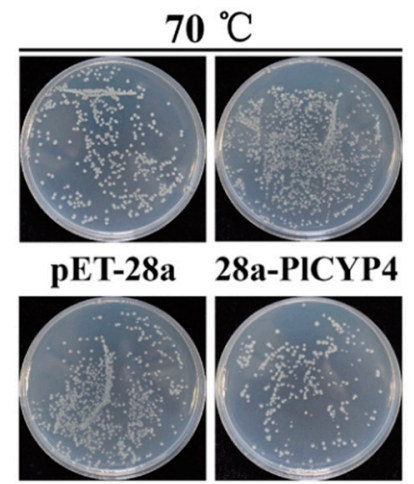

28a-PICYP6 28a-PICYP6

(T-a)

(T-b)

$\mathbf{B}$

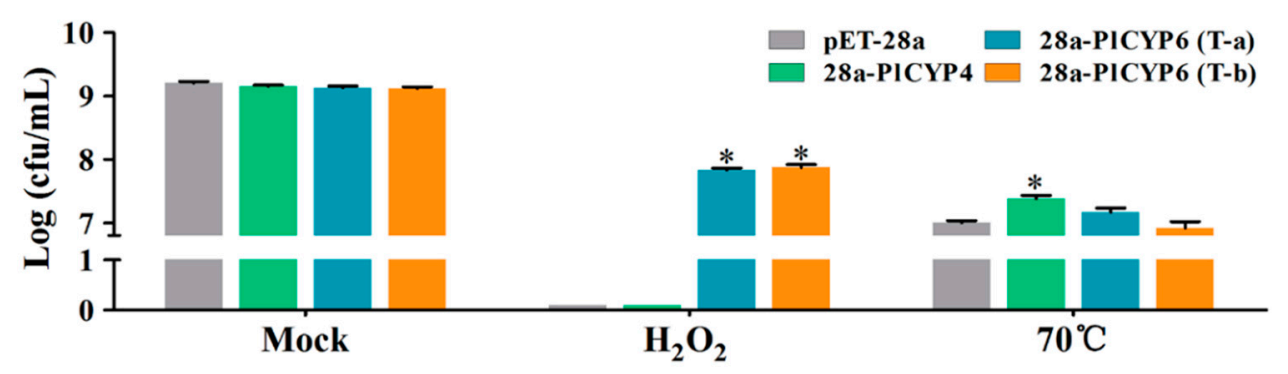

Figure 7. Assay of $\mathrm{PlCYPs}$ ' response to different abiotic stresses. (A) Growth analysis of E. coli cells containing an empty vector (pET-28a) and recombinant vectors (pET-PlCYP4 and pET-PICYP6) was carried on Luria-Bertani (LB) liquid medium with two treatments (supplemented with $1 \mathrm{mM} \mathrm{H}_{2} \mathrm{O}_{2}$ and $70{ }^{\circ} \mathrm{C}$ for $20 \mathrm{~min}$ ). The treated cultures were diluted to a suitable concentration and then spread onto the LB plate to count the number of living bacteria. (B) Statistical analysis of the bacterial colonies. The error bar represents the SD of three replicates. * denotes $p<0.01$.

\section{Discussion}

P. lilacinum has a high efficiency in terms of parasitizing nematode eggs and is beneficial for plant growth, making it an effective biocontrol agent for controlling plant parasitic nematodes. However, there are obstacles in the large-scale application of this fungal agent, such as the difficulty in maintaining its activity and its adaption to unfavorable environments, which lead to a short shelf life and unstable field biocontrol effect. Understanding the molecular mechanisms of nematode parasitism and adaption to adversity will promote the application of this fungus in plant protection. The current study revealed the composition of members of the Cyclophilin gene family in P. lilacinum by profiling their expression patterns during development and nematode egg infection and analyzing their potential functions. These results provide a framework for future studies and the use of CYPs in P. lilacinum.

In the present study, $10 \mathrm{PlCY} P$ s were identified through a genome-wide analysis. Within the fungi analyzed in this study, the Ascomycotina fungi were shown to have approximately 10 CYP proteins, whereas Saccharomycotina such as C. albicans, C. glabrata, and S. cerevisiae have 6, 6, and 8 CYPs, respectively, supporting that Saccharomycotina has a lower number of CYPs than Ascomycotina [38]. In addition, previous research showed that prokaryotes generally have less CYPs than eukaryotes [14].

Phylogenetic analysis of 10 PICYPs in P. lilacinum with 84 CYPs identified in nine Ascomycota fungi, and generated a tree that was artificially divided into 10 groups. The phylogenetic tree did not show a close relationship within the 10 PICYPs, which were distributed into separated groups with no paralog. This is probably associated with the functional diversity of the PlCYPs. In addition, three groups G, H, and I were found to possess all of the analyzed species, while the CYPs of Ascomycotina and Saccharomycotina fungi were clearly separated into two branches, suggesting evolutionary 
divergence between the two kinds of fungi. Motif and gene structure analysis is an effective tool that can be employed to understand sequence signatures in plant research [39-41]. The motif distributions and gene structures of fungal CYPs associated with the phylogenetic tree were exhibited. We found that fungal CYPs in the same group had similar motif arrangements, and six motifs (motifs 1-5 and 7) were annotated as having the conserved CLD, similar to the CYPs in plants. In particular, members in group B, such as PICYP5 were found to lack motifs 2 and 5, suggesting that proteins in this group may possess a degenerated CLD with a weak catalytic capacity. Overall, the motifs and gene structure analyses provided additional evidence to support the phylogenetic relationship.

The cyclophilin-like domains in the PICYPs sequences were highly conserved, which is consistent with the previous description [42]. However, the PlCYPs varied widely in terms of sequence length, isoelectric point, and subcellular localization, providing another piece of evidence for functional diversity of PICYPs. In the PPIase superfamily, the CYP family is the only one that has members which localize in the mitochondria [42]. However, the subcellular localization of PlCYPs did not show a close relationship with the phylogenetic analysis. For instance, both PICYP5 and PICYP7 were predicted to localize in the nucleus, but PICYP5 has an RRM domain and was classified into group B, and PICYP7 which does not harbor an RRM domain and was placed into group A. This indicates that PICYPs in the same cellular place play different roles, supporting a previous observation that it is insufficient to regard localization as the sole criteria for classification of CYPs [21].

The expression profiles of PlCYPs in different fungal morphologies, during the process of egg parasitism and under abiotic stresses, indicated that the 10 PlCYPs appear to have a wide range of diverse roles within the cell. Previous studies have demonstrated that plant CYPs have tissue-specific expression [23]. In our results, PICYPs showed a ubiquitous expression abundance at different stages of fungal development, which is different from plant CYPs. In addition, we measured the expressions of PlCYPs at different time points after egg inoculation, and the results confirmed the transcriptome data (Figure S6), demonstrating that the expressions of all PlCYPs were up-regulated to varying degrees. PlCYP5 was significantly expressed at $0.5 \mathrm{hpi}$, suggesting PICYP5 might have a similar function as its orthologs AtCYP59 and SpRct1 (Table S2) that are involved in transcriptional regulation $[35,36]$. The expression of $P l C Y P 3$ was increased significantly after 3 hpi, suggesting that $P l C Y P 3$ may assist in the eggshell invasion of hyphae. In addition, other genes, such as PlCYP2, 6, 8, and 9, showed significant expression levels at later stages of inoculation, indicating their potential roles in egg parasitism.

It has been shown that CYP proteins play crucial roles in abiotic stress tolerance [22,23]. For $P I C Y P S$, the long- and short-term responses to abiotic stresses were measured. There were differences between the two results, suggesting different mechanisms of response. The expression of PlCYP5 increased during short-term responses to stresses but decreased in long-term responses, implying that it may be involved in early-stage regulation of genes related to the stress response. Nevertheless, PlCYP4 exhibited a higher expression level in both the short- and long-term responses to high temperature. Similarly, $P l C Y P 6$ had a higher expression level in both short- and long-term response to $\mathrm{H}_{2} \mathrm{O}_{2}$. This indicates that PlCYP4 and PlCYP6 may be related to high temperature and $\mathrm{H}_{2} \mathrm{O}_{2}$ stress tolerance. In addition, the related functions of PICYP4 and PICYP6 were further proved by the heterologous expression assay. This study indicates the potential functions of PlCYPs in pathogenicity and the abiotic stress responses, and also provides a frame for further analysis of the CYP gene family in Ascomycotina fungi. However, the accurate functions of PICYPs in P. lilacinum need to be explored in the future.

\section{Materials and Methods}

\subsection{Fungal Strains and Growth Condition}

The fungus P. lilacinum strain 36-1 was isolated from the egg surface of Meloidogyne incognita from field soil from Hubei Province in China. Strain 36-1 was cultured on a potato dextrose agar (PDA) plate 
at $28^{\circ} \mathrm{C}$ for normal culture and on Czapek-Dox medium $(\mathrm{CZM})$ at $28^{\circ} \mathrm{C}$ for phenotypic determination. It was stored at $4{ }^{\circ} \mathrm{C}$.

\subsection{Identification and Classification of CYP Genes in P. lilacinum Strain 36-1}

To identify $C Y P$ genes in the genome of P. lilacinum strain 36-1, a local BLASTP search was performed using the amino acid sequences of hCYPA (GenBank: AAI37059.1). The generated sequences were searched again for other PICYPs until no new sequence appeared. ScanProsite (https://prosite.expasy.org/) and the Conserved Domain Database (CDD) were used to ensure that the candidate PlCYPs harbored a cyclophilin-like domain (CLD). The genome of the P. lilacinum strain PLFJ-1 (Accession: LSBI00000000) was regarded as another database for validation, using the same searching procedure.

The coding sequences of PICYPs were obtained by comparing the genomic and transcriptome databases [10]. The molecular weight $(\mathrm{kD})$ and isoelectric point $(\mathrm{pI})$ of each protein were predicted by Expasy programs using amino acid sequences (http://www.expasy.org/tools/). The WoLF PSORT II program (https://www.genscript.com/wolf-psort.html) was used for the prediction of subcellular localizations of PICYPs.

\subsection{Protein Alignment and Phylogenetic Analysis}

The full-length coding sequences of PlCYPs were aligned by the ClusterW program with default parameters. To show the residual variation of CLD, sequences of the CLD were singled out for alignment in the same manner. For phylogenetic analysis, amino acid sequences of the fungal CYPs were downloaded from National Center for Biotechnology Information (NCBI, Rockville Pike, Bethesda MD, USA) by accession number (Table S3), and the sequences were aligned using the MUSCLE program of MEGA 6.0 with default settings. MEGA 6.0 software (http://www.megasoftware.net/) was employed to construct the phylogenetic tree using the neighbor-joining (NJ) method with 1000 bootstrap replicates. The tree was exported into the Interactive Tree of Life (http://itol.embl.de) for further annotation.

\subsection{Motifs and Gene Structure Analyses}

The MEME (http://meme-suite.org/tools/meme) was used to analyze conserved and potential motifs with the parameter settings: any number of repetitions, a minimum motif width of 6 , a maximum motif width of 200, and a maximum number of motifs of 20. Subsequently, the created motifs were annotated by the Pfam (http://pfam.xfam.org/). Exon-intron organization was predicted using Gene Structure Display Server 2.0 (GSDS 2.0) (http://gsds.cbi.pku.edu.cn/) by comparing the coding sequences with corresponding DNA sequences.

\subsection{Transient Expression of Tobacco}

The open reading frames of $P l C Y P 5$ and $P l C Y P 7$ were cloned into the vector pEGAD to generate the vectors pEGAD::PICYP5 and PEGAD::PICYP7 [43]. The recombinant vectors were transferred into Agrobacterium tumefaciens GV3101 by electroporation. The cultured A. tumefaciens cells $\left(\mathrm{OD}_{600}=0.4\right)$ were infiltrated into the leaves of Nicotiana benthamiana which were grown in the greenhouse for four weeks at $26^{\circ} \mathrm{C}$ under a $16 \mathrm{~h} \mathrm{light} / 8 \mathrm{~h}$ dark cycle. The same process using the empty vector $\mathrm{pEGAD}$ was used as a control. Subcellular localizations of the fused proteins were visualized using fluorescence microscopy at $48 \mathrm{~h}$ after infiltration.

\subsection{Sample Treatments for $q R T-P C R$}

Different fungal morphological samples were collected from P. lilacinum strain 36-1, including conidia, germinating conidia, blastospores, vegetative mycelia, and aerial mycelia. Briefly, the conidia were washed from P. lilacinum strain 36-1 that had been cultured on PDA for three weeks. For germination, the conidia were standardized to $1 \times 10^{7}$ conidia per milliliter and added to liquid 
CZM with a dilution ratio of 1:100 for shaking culture, and the samples were then collected at 4 and $8 \mathrm{~h}$ post inoculation (hpi). To produce blastospores, we conducted the study based on previously reported methods for B. bassiana with modifications; namely, potato dextrose broth (PDB) was used instead of Sabouraud's medium [44]. Vegetative and aerial mycelia were separated and collected from the fungus that was cultured for one week on PDA. All collected samples were immediately used for RNA extraction.

Mycelia-infecting nematode eggs were collected by a previously described method [10]. Specifically, $M$. incongnita egg-masses were isolated from tomato roots and suspended in sterile distilled water after being sterilized with $0.5 \%(v / v) \mathrm{NaClO}$. The conidia of P. lilacinum strain $36-1$ were pre-cultured in liquid minimal media for $24 \mathrm{~h}$ and washed by sterile water. Then, the pre-cultured conidia were mixed with M. incongnita eggs. Mycelia were collected at 0, 0.5, 1, 3, 6, and 12 hpi by filtering.

In the abiotic stress treatment assay, the conidia were standardized to $1 \times 10^{5}$ conidia per $\mathrm{mL}$, and $50 \mu \mathrm{L}$ of conidia was spread on CZM supplemented with $1 \mathrm{M} \mathrm{NaCl}, 1.2 \mathrm{M}$ sorbitol, $5 \mathrm{mM} \mathrm{H}_{2} \mathrm{O}_{2}$, or $0.15 \mathrm{mg} / \mathrm{mL}$ Congo red, and the $\mathrm{pH}$ was adjusted to 10. Strain 36-1 cultured on normal CZM was regarded as the control. The mycelia of the strain 36-1 were collected at $3 \mathrm{dpi}$. To test the expressions of PICYPs under different temperatures, the P. lilacinum 36-1 strains were cultured on normal CZM for three days, and were then were cultured at 4 or $35^{\circ} \mathrm{C}$ for $1 \mathrm{~h}$.

A liquid assay was also performed to detect the short-term expressions of PlCYPs under the same abiotic stress conditions. The conidia were inoculated in $150 \mathrm{~mL}$ liquid CZM and shock culture for two days. After filtration, the collected mycelia were immediately transferred to the fresh liquid CZM containing the same abiotic stresses for further shaking culture. The mycelia were collected at 15, 30, 45 , and $60 \mathrm{~min}$. All of the above-mentioned assays were performed with three biological replicates.

\subsection{RNA Extraction and $q R T-P C R$}

Total RNA was isolated with TRIzol reagent (Invitrogen ${ }^{\mathrm{TM}}$, Carlsbad, CA, USA) according to the manufacturer's protocols. The total RNA was treated with the DNA-free ${ }^{\mathrm{TM}}$ DNA Removal Kit (Invitrogen ${ }^{\mathrm{TM}}$, Carlsbad, CA, USA), and was then used to generate the first strand of cDNA with the RevertAid First Strand cDNA Synthesis Kit (Thermo Scientific, Rockford, IL, USA). Primers used for qRT-PCR are listed in Table S4. The amplification efficiencies were individually verified and are shown in Figure S7. Gene expression abundance was analyzed by qRT-PCR using the Bio-Rad CFX96 Real Time System and SsoFast ${ }^{\mathrm{TM}}$ EvaGreen Supermix (Bio-Rad, Hercules, CA, USA). The fold changes of gene expression were calculated versus the control by the $2^{-\Delta \Delta C t}$ method (Table S5), which was applied to draw a heat map using the pheatmap function in R. $\beta$-actin and tubulin were used as endogenous reference genes $[9,10]$.

\subsection{Phenotypic Assay of PlCYP4 and PlCYP6 in E. coli}

Heterologous expressions of PlCYP4 and PlCYP6 in E. coli were carried out to detect whether these genes enhance the tolerance of $E$. coli to abiotic stress. The pET-28a $(+)$ plasmid was used as the original vector and the full-length CDS fragments of selected PlCYP genes were inserted between restriction sites BamH I and EcoR I to generate recombinant expression vectors. Rosetta (DE3) cells containing the recombinant vectors or an empty vector were grown in $10 \mathrm{~mL}$ of Luria-Bertani (LB) medium with $50 \mu \mathrm{g} / \mathrm{mL}$ kanamycin until the $\mathrm{OD}_{600}$ was about 0.6 . A final concentration of $0.5 \mathrm{mmol} / \mathrm{L}$ $\beta$-d-thiogalactopyranoside (IPTG) was then added into the medium and E. coli cells were continually shaken at $16^{\circ} \mathrm{C}$ and $150 \mathrm{rpm}$ for $18 \mathrm{~h}$. Finally, all samples were adjusted to $\mathrm{OD}_{600}=1.0$ and then further diluted to $10^{-1}, 10^{-2}$, and $10^{-3}$. In total, $2 \mu \mathrm{L}$ of each dilution was spotted on LB plates containing $600 \mathrm{mM} \mathrm{NaCl}, 800 \mathrm{mM}$ sorbitol or $1 \mathrm{mM} \mathrm{H}_{2} \mathrm{O}_{2}$, and LB (pH 10).

Overnight, the transformants were grown overnight in LB liquid medium containing $50 \mu \mathrm{g} / \mathrm{mL}$ kanamycin, and then all samples were diluted to $\mathrm{OD}_{600}=0.1$. In total, a $10 \mu \mathrm{L}$ aliquot of solution was added into $10 \mathrm{~mL}$ of LB liquid medium that contained $0.5 \mathrm{mM} \mathrm{IPTG}$ and $1 \mathrm{mM} \mathrm{H}_{2} \mathrm{O}_{2}$. This was shaken at $37^{\circ} \mathrm{C}$ and $180 \mathrm{rpm}$ for $12 \mathrm{~h}$. The strains cultured in $\mathrm{LB}$ without $\mathrm{H}_{2} \mathrm{O}_{2}$ were used as controls. 
In addition, partial cultures of the control group were treated at $70^{\circ} \mathrm{C}$ for $20 \mathrm{~min}$. The above-mentioned assays were performed with three replicates.

\subsection{Statistical Analysis}

Data quantified from the qRT-PCR of three biological replicates were subjected to two-way ANOVA analysis, followed by Bonferroni's post-test for data comparison. A $p$-value of less than 0.01 was deemed to represent a significant difference.

Supplementary Materials: The following are available online at http://www.mdpi.com/1422-0067/20/12/2978/s1.

Author Contributions: Y.X. and X.X. designed this study. C.M. and C.X. carried out the bioinformatics analyses. C.M. and C.X. performed the qRT-PCR experiments. J.L. and Q.H. helped in the phenotype experiments. C.M. wrote the manuscript. Y.X., X.X. and G.W. helped in discussions of the manuscript. All authors read and approved the final manuscript.

Funding: This research was funded by the national natural science foundation of China, grant number 31872019 and national key R\&D program of China, grant number 2018YFD0200500, 2018YFD0201000

Conflicts of Interest: The authors declare no conflict of interest.

\section{References}

1. Abad, P.; Gouzy, J.; Aury, J.; Castagnone-Sereno, P.; Danchin, E.G.J.; Deleury, E.; Caillaud, M.C. Genome sequence of the metazoan plant-parasitic nematode Meloidogyne incognita. Nat. Biotechnol. 2008, 26, 909-915. [CrossRef] [PubMed]

2. Jones, J.T.; Haegeman, A.; Danchin, E.G.J.; Gaur, H.S.; Helder, J.; Jones, M.G.K.; Perry, R.N. Top 10 plant-parasitic nematodes in molecular plant pathology. Mol. Plant Pathol. 2013, 14, 946-961. [CrossRef] [PubMed]

3. Atkins, S.D.; Clark, I.M.; Pande, S.; Hirsch, P.R.; Kerry, B.R. The use of real-time PCR and species-specific primers for the identification and monitoring of Paecilomyces lilacinus. FEMS Microbiol. Ecol. 2005, 51, 257-264. [CrossRef]

4. Anastasiadis, I.A.; Giannakou, I.O.; Prophetou-Athanasiadou, D.A.; Gowen, S.R. The combined effect of the application of a biocontrol agent Paecilomyces lilacinus, with various practices for the control of root-knot nematodes. Crop Prot. 2008, 27, 352-361. [CrossRef]

5. Huang, W.K.; Cui, J.K.; Liu, S.M.; Kong, L.A.; Wu, Q.S.; Peng, H.; Peng, D.L. Testing various biocontrol agents against the root-knot nematode (Meloidogyne incognita) in cucumber plants identifies a combination of Syncephalastrum racemosum and Paecilomyces lilacinus as being most effective. Biol. Control 2016, 92, 31-37. [CrossRef]

6. Hajji, L.; Hlaoua, W.; Regaieg, H.; Horrigue-Raouani, N. Biocontrol potential of Verticillium leptobactrum and Purpureocillium lilacinum Against Meloidogyne javanica and Globodera pallida on Potato (Solanum tuberosum). Am. J. Patato Res. 2017, 94, 178-183. [CrossRef]

7. Kiewnick, S.; Neumann, S.; Sikora, R.A.; Frey, J.E. Effect of Meloidogyne incognita inoculum density and application rate of Paecilomyces lilacinus strain 251 on biocontrol efficacy and colonization of egg masses analyzed by real-time quantitative PCR. Phytopathology 2011, 101, 105-112. [CrossRef]

8. Prasad, P.; Varshney, D.; Adholeya, A. Whole genome annotation and comparative genomic analyses of bio-control fungus Purpureocillium lilacinum. BMC Genom. 2015, 16, 1004. [CrossRef]

9. Wang, G.; Liu, Z.G.; Lin, R.M.; Li, E.F.; Mao, Z.C.; Ling, J.; Xie, B. Biosynthesis of antibiotic leucinostatins in bio-control fungus purpureocillium lilacinum and their inhibition on Phytophthora revealed by genome mining. PloS Pathog. 2016, 12, e1005685. [CrossRef]

10. Xie, J.L.; Li, S.J.; Mo, C.M.; Xiao, X.Q.; Peng, D.L.; Wang, G.F.; Xiao, Y. Genome and transcriptome sequences reveal the specific parasitism of the nematophagous Purpureocillium lilacinum 36-1. Front. Microbiol. 2016, 7, 1084. [CrossRef]

11. Handschumacher, R.E.; Harding, M.W.; Rice, J.; Drugge, R.J.; Speicher, D.W. Cyclophilin: A specific cytosolic binding protein for cyclosporin A. Science 1984, 226, 544-547. [CrossRef] [PubMed]

12. Gothel, S.F.; Marahiel, M.A. Peptidyl-prolyl cis-trans isomerases, a superfamily of ubiquitous folding catalysts. Cell. Mol. Life Sci. 1999, 55, 423-436. [CrossRef] [PubMed] 
13. Schiene-Fischer, C. Multidomain peptidyl prolyl cis/trans isomerases. Biochim. Biophys. Acta Gen. Subj. 2014, 1850, 2005-2016. [CrossRef] [PubMed]

14. Hayano, T.; Takahashi, N.; Kato, S.; Maki, N.; Suzuki, M. Two distinct forms of peptidylprolyl-cis-trans-isomerase are expressed separately in periplasmic and cytoplasmic compartments of Escherichia coli cells. Biochemistry 1991, 30, 3041-3048. [CrossRef] [PubMed]

15. Andrzej, G. Peptidylprolyl cis/trans isomerases (immunophilins): Biological diversity-targets-functions. Curr. Top. Med. Chem. 2003, 3, 1315-1347. [CrossRef]

16. Liu, J.; Farmer, J.D.; Lane, W.S.; Friedman, J.; Weissman, I.; Schreiber, S.L. Calcineurin is a common target of cyclophilin-cyclosporin A and FKBP-FK506 complexes. Cell 1991, 66, 807-815. [CrossRef]

17. Pemberton, T.J.; Kay, J.E. Identification and comparative analysis of the peptidyl-prolyl cis/trans isomerase repertoires of H. sapiens, D. melanogaster, C. elegans, S. cerevisiae and Sz. pombe. Comp. Funct. Genom 2005, 6, 277-300. [CrossRef] [PubMed]

18. Gan, P.H.P.; Shan, W.X.; Blackman, L.M.; Hardham, A.R. Characterization of cyclophilin-encoding genes in Phytophthora. Mol. Genet. Genom. 2009, 281, 565-578. [CrossRef]

19. Arevalo-Rodriguez, M.; Wu, X.; Hanes, S.D.; Heitman, J. Prolyl isomerases in yeast. Front. Biosci. 2004, 9, 2420-2446. [CrossRef]

20. Dolinski, K.; Muir, S.; Cardenas, M.; Heitman, J. All cyclophilins and FK506 binding proteins are, individually and collectively, dispensable for viability in Saccharomyces cerevisiae. Proc. Natl. Acad. Sci. USA 1997, 94, 13093-13098. [CrossRef]

21. Trivedi, D.K.; Yadav, S.; Vaid, N.; Tuteja, N. Genome wide analysis of Cyclophilin gene family from rice and Arabidopsis and its comparison with yeast. Plant Signal. Behav. 2012, 7, 1653-1666. [CrossRef] [PubMed]

22. Mainali, H.R.; Chapman, P.; Dhaubhadel, S. Genome-wide analysis of Cyclophilin gene family in soybean (Glycine max). BMC Plant Biol. 2014, 14, 282. [CrossRef] [PubMed]

23. Wang, Q.Q.; Wang, Y.; Chai, W.B.; Song, N.N.; Wang, J.; Cao, L.M.; Li, X. Systematic analysis of the maize cyclophilin gene family reveals ZmCYP15 involved in abiotic stress response. Plant Cell Tissue Organ Cult. 2017, 128, 543-561. [CrossRef]

24. Anna, K.; Janine, B.; Anne-Marie, K.; Jean, C.; Maria, J.D.; Catherine, K. KIN241: A gene involved in cell morphogenesis in Paramecium tetraurelia reveals a novel protein family of cyclophilin-RNA interacting proteins (CRIPs) conserved from fission yeast to man. Mol. Microbiol. 2001, 42, 257-267. [CrossRef]

25. Duina, A.A.; Chang, H.C.; Marsh, J.A.; Lindquist, S.; Gaber, R.F. A cyclophilin function in Hsp90-dependent signal transduction. Science 1996, 274, 1713-1715. [CrossRef] [PubMed]

26. Horowitz, D.S.; Lee, E.J.; Mabon, S.A.; Misteli, T. A cyclophilin functions in pre-mRNA splicing. EMBO J. 2002, 21, 470-480. [CrossRef] [PubMed]

27. Shaw, P.E. Peptidyl-prolyl isomerases: A new twist to transcription. EMBO Rep. 2002, 3, 521-526. [CrossRef] [PubMed]

28. Kumari, S.; Singh, P.; Singla-Pareek, S.L.; Pareek, A. Heterologous expression of a salinity and developmentally regulated rice cyclophilin gene (OsCyp2) in E. coli and S. cerevisiae confers tolerance towards multiple abiotic stresses. Mol. Biotechnol. 2009, 43, 195-204. [CrossRef]

29. Ruan, S.L.; Ma, H.S.; Wang, S.H.; Fu, Y.P.; Xin, Y.; Liu, W.Z.; Chen, H.Z. Proteomic identifcation of OsCYP2, a rice cyclophilin that confers salt tolerance in rice (Oryza sativa L.) seedlings when overexpressed. BMC Plant Biol. 2011, 11, 1117-1120. [CrossRef]

30. Trivedi, D.K.; Ansari, M.W.; Tuteja, N. Multiple abiotic stress responsive rice cyclophilin: (OsCYP-25) mediates a wide range of cellular responses. Commun. Integr. Biol. 2013, 6, e25260. [CrossRef]

31. Kim, S.K.; You, Y.N.; Park, J.C.; Joung, Y.; Kim, B.G.; Ahn, J.C.; Cho, H.S. The rice thylakoid luminal cyclophilin OsCYP20-2 confers enhanced environmental stress tolerance in tobacco and Arabidopsis. Plant Cell Rep. 2012, 31, 417-426. [CrossRef] [PubMed]

32. Chu, Z.J.; Sun, H.H.; Ying, S.H.; Feng, M.G. Vital role for cyclophilin B (CypB) in asexual development, dimorphic transition and virulence of Beauveria bassiana. Fungal Genet. Biol. 2017, 105, 8-15. [CrossRef]

33. Viaud, M.C.; Balhadere, P.V.; Talbot, N.J. A Magnaporthe grisea cyclophilin acts as a virulence determinant during plant infection. Plant Cell 2002, 14, 917-930. [CrossRef] [PubMed]

34. Viaud, M.; Brunet-Simon, A.; Brygoo, Y.; Pradier, J.M.; Levis, C. Cyclophilin A and calcineurin functions investigated by gene inactivation, cyclosporin A inhibition and cDNA arrays approaches in the phytopathogenic fungus Botrytis cinerea. Mol. Microbiol. 2003, 50, 1451-1465. [CrossRef] [PubMed] 
35. Gullerova, M.; Barta, A.; Lorkovic, Z.J. AtCyp59 is a multidomain cyclophilin from Arabidopsis thaliana that interacts with SR proteins and the C-terminal domain of the RNA polymerase II. RNA 2006, 12, 631-643. [CrossRef] [PubMed]

36. Gullerova, M.; Barta, A.; Lorkovic, Z.J. Rct1, a nuclear RNA recognition motif-containing cyclophilin, regulates phosphorylation of the RNA polymerase II C-terminal domain. Mol. Cell. Biol. 2007, 27, 3601-3611. [CrossRef]

37. Zydowsky, L.D.; Etzkorn, F.A.; Chang, H.Y.; Ferguson, S.B.; Stolz, L.A.; Ho, S.; Walsh, C.T. Active-site mutants of human cyclophilin-a separate peptidyl-prolyl isomerase activity from cyclosporine-a binding and calcineurin inhibition. Protein Sci. 1992, 1, 1092-1099. [CrossRef] [PubMed]

38. Pemberton, T.J. Identification and comparative analysis of sixteen fungal peptidyl-prolyl cis/trans isomerase repertoires. BMC Genom. 2006, 7, 244. [CrossRef]

39. Han, Y.H.; Ding, T.; Su, B.; Jiang, H.Y. Genome-wide identification, characterization and expression analysis of the chalcone synthase family in maize. Int. J. Mol. Sci. 2016, 17, 161. [CrossRef]

40. Wang, G.; Wang, T.; Jia, Z.H.; Xuan, Z.P.; Pan, D.L.; Guo, Z.R.; Zhang, J.Y. Genome-wide bioinformatics analysis of MAPK gene family in kiwifruit (Actinidia chinensis). Int. J. Mol. Sci. 2018, 19, 2510. [CrossRef]

41. Li, J.B.; Zhang, J.; Jia, H.X.; Yue, Z.Q.; Lu, M.Z.; Xin, X.B.; Hu, J.J. Genome-wide characterization of the $s H s p$ gene family in Salix suchowensis reveals its functions under different abiotic stresses. Int. J. Mol. Sci. 2018, 19, 3246. [CrossRef] [PubMed]

42. Wang, P.; Heitman, J. The cyclophilins. Genome Biol. 2005, 6, 226. [CrossRef] [PubMed]

43. Cutler, S.R.; Ehrhardt, D.W.; Griffitts, J.S.; Somerville, C.R. Random GFP.:cDNA fusions enable visualization of subcellular structures in cells of Arabidopsis at a high frequency. Proc. Natl. Acad. Sci. USA 2000, 97, 3718-3723. [CrossRef] [PubMed]

44. Ying, S.H.; Feng, M.G. Novel blastospore-based transformation system for integration of phosphinothricin resistance and green fluorescence protein genes into Beauveria bassiana. Appl. Microbiol. Biotechnol. 2006, 72, 206-210. [CrossRef]

(C) 2019 by the authors. Licensee MDPI, Basel, Switzerland. This article is an open access article distributed under the terms and conditions of the Creative Commons Attribution (CC BY) license (http://creativecommons.org/licenses/by/4.0/). 